\title{
Dynamic response of structures constructed from smart materials
}

\section{T K Caughey}

\author{
California Institute of Technology, Pasadena, CA, USA
}

Received 1 January 1994, accepted for publication 1 March 1994

\begin{abstract}
The Dynamic analysis of structures constructed of homogeneous smart materials is greatly simplified by the observation that the eigenfunctions of such structures are identical to those of the same structures constructed entirely of purely elastic materials. The dynamic analysis of such structures is thus reduced to the analysis of the temporal behaviour of the eigenmodes of the structure. The theory is illustrated for both continuous and discrete structures using the generalization of 'positive position feedback' to distributed control.
\end{abstract}

\section{Introduction}

In the last decade the introduction of 'smart' materials has made possible the robust control of multi-degree-offreedom and continuous structures. If the structure is fabricated of homogeneous 'smart' material the dynamic analysis of the structure is greatly simplified by the observation that the eigen functions of such structures are identical to those of the same structure constructed entirely of purely elastic materials. The dynamic analysis of such structures is thus reduced to the temporal analysis of the eigenmodes of the structure.

\section{Background}

Consider the following partial differential equation with homogeneous boundary conditions and given initial conditions:

$$
A(x) U_{t t}+D_{1}\left(B(x) D_{1} U\right)=f(x, t): 0<x<L .
$$

In equation (1) $A(x)$ is a mass density, $B(x)$ a distribution of elastic stiffness, $D_{1}$ a spatial operator, $f(x, t)$ a spatial distribution of time varying force. If equation (1) has homogeneous boundary conditions, then under weak restrictions on $A(x), B(x)$ and $D_{1}$ equation (1) possesses a complete set of eigenvalues $\omega_{\imath}^{2}$ and corresponding orthonormal eigenfunctions $\phi_{i}(x)$.

The solution of equation (1) can therefore be express in the form

$$
(x, t)=\sum_{i=0}^{\infty} a_{i}(t) \phi_{i}(x)
$$

where $a_{i}(t), i \in[1, \infty)$ are the solutions of the ordinary differential equations

$$
\ddot{a}_{i}+\omega_{i}^{2} a_{i}=q_{i}(t)
$$

with given initial conditions $a_{0}(t), \dot{a}_{0}(t)$, and $q_{i}(t)$ is given by:

$$
q_{i}(t)=\int_{0}^{L} f(x, t) \phi_{i}(x) \mathrm{d} x .
$$

Consider now the following partial differential with homogeneous boundary conditions and given initial conditions

$$
A(x) U_{t t}+D_{1}\left(B(x) D_{1} V\right)=f(x, t): 0<x<L
$$

where $V(x, t)$ is given by

$$
V(x, t)=\int_{-\infty}^{t} h(t-\tau) U(x, \tau) \mathrm{d} \tau
$$

or

$$
V(x, t)=\mathcal{H} U(x, t)
$$

where $\mathcal{H}$ is the hereditary operator relating $V(x, t)$ and $U(x, t)$ such as occurs in viscoelasticity.

As in the case of equation (1) we seek a solution in the form

$$
U(x, t)=\sum_{i=0}^{\infty} b_{i}(t) \phi_{i}(x)
$$

where $b_{i}(t), i \in[1, \infty)$ are the solutions of the integrodifferential equations:

$$
\ddot{b}_{i}+\omega_{i}^{2} \mathcal{H} b_{i}=q_{i}(t)
$$

with given initial conditions $b_{i}(0), \dot{b}_{i}(0)$, and $q_{i}(t)$ given by equation (4).

Equation (9) is easily solved using integral transform methods such as Laplace or Fourier. This technique was used by Caughey (1962) to study the dynamics of systems with linear hysteretic damping. 


\section{Active damping control}

All physical systems possess some degree of internal damping which usually increases with frequency, so that the higher-frequency modes are more highly damped than the low-frequency modes. Flexible space structures tend to have very light damping in the low-frequency modes while the high-frequency modes are usually adequately damped. This means that the settling time for flexible space structures can be quite long unless active damping techniques are employed to increase the effective damping. In addition to the problem of settling time, light damping in the low-frequency modes makes the structure very sensitive to disturbances. For these reasons there has been considerable research into the active control of structures. Initially these studies were concerned with aerospace structures, but in recent years such studies have been extended to civil structures such as buildings and bridges. Numerous control techniques have been suggested; these have been reviewed by Balas (1982) and Meirovitch $e t$ al (1981). Most techniques employ the concept of optimal control, but are often plagued by the problem of observation spillover. This tends to destabilize some of the uncontrolled or unmodelled modes, as has been amply demonstrated by Balas (1978a) and Schaechter (1982). Another important problem which has been largely ignored is the problem of actuator dynamics. This poblem has been considered by Balas (1978b) and Caughey and Goh (1982, Goh and Caughey 1985), who have shown that the finite bandwidth of practical actuators, while providing excellent control of low-frequency modes, may destabilize the intermediate or higher-frequency modes.

The technique of collocated direct velocity feedback has been examined by Auburn (1980), Balas (1978b) and Chen (1982) and shown to be unconditianally stable in the absence of actuator dynamics. However if actuator dynamics are included, instabilities may arise unless special precautions are taken. It is possible to design stable velocity feedback control systems, including actuator dynamics, and several techniques are discussed by Caughey and Goh (1982). An alternate control scheme first proposed by Caughey in the late 1970's as a way in which collocated sensors and actuators could be used in active control to increase the damping of the lower modes of space structures without the actuator dynamics causing instability in the uncontrolled or unmodelled modes. Caughey and his students Goh and Fanson further developed this rather unconventional technique, which is a generalization of the concept of the tuned vibration absorber. The theory of positive position feedback is covered in the papers by Goh and Caughey $(1983,1985)$ and experiments with the technique are covered in the paper by Fanson and Caughey (1987). Addition details are contained in two Calech Ph.D. thesis Goh (1983) and Fanson (1987). The technique has been exploited by Fanson at JPL and is now covered by a NASA patent.

In addition to its other advantages, positive position feedback can, in concept, be incorporated directly into the material of which the structure is fabricated, hence the title of this paper.

\section{Positive position feedback}

\subsection{Single degree of freedom case}

Consider the following system of differential equations describing a system to be controlled and the dynamics of its associated actuator:

$$
\begin{aligned}
& m_{1} \ddot{x}+d_{1} \dot{x}+k_{1} x=k_{\mathrm{c}} y+f(t) \\
& m_{\mathrm{a}} \ddot{y}+d_{\mathrm{a}} \dot{y}+k_{\mathrm{a}} y=k_{\mathrm{a}} x
\end{aligned}
$$

It will be observed that if in equation (10) $k_{\mathrm{c}}=k_{\mathrm{a}}$ and $k_{1}=k+k_{a}$, then equations(10) and (11) describe the conventional tuned vibration absorber. Thus positive position feedback may be regarded as a generalization of the well known tuned vibration absorber.

Define:

$$
\begin{aligned}
& \omega_{\mathrm{a}}^{2}=\frac{k_{\mathrm{a}}}{m_{\mathrm{a}}} \\
& \beta_{\mathrm{a}}=\frac{d_{\mathrm{a}}}{m_{\mathrm{a}}} \\
& \omega_{\mathrm{ad}}^{2}=\omega_{\mathrm{a}}^{2}-\frac{\beta_{\mathrm{a}}^{2}}{4} \\
& \omega_{1}^{2}=\frac{k_{1}}{m_{1}} \\
& \beta_{1}=\frac{d_{1}}{m_{1}} \\
& \lambda=\frac{k_{\mathrm{c}}}{k_{1}}
\end{aligned}
$$

Equations (10) and (11) take the form

$$
\begin{aligned}
& \ddot{x}+\beta_{\mathrm{I}} \dot{x}+\omega_{\mathrm{1}}^{2} x=\lambda \omega_{1}^{2} y+\frac{f(t)}{m_{1}} \\
& \ddot{y}+\beta_{\mathrm{a}} \dot{y}+\omega_{\mathrm{a}}^{2} y=\omega_{\mathrm{a}}^{2} x
\end{aligned}
$$

Equations (13) and (14) may combined in the form

$$
\begin{aligned}
& \ddot{x}+\beta_{1} \dot{x}+\omega_{1}^{2} \mathcal{H} x=\frac{f(t)}{m_{1}} \\
& \mathcal{H}=1-\lambda \mathcal{H}_{\mathrm{a}} \\
& \mathcal{H}_{\mathrm{a}} x=\int_{-\infty}^{t} h(t-\tau) x(\tau) \mathrm{d} \tau=y(t) \\
& h(t)=\exp \left(-\frac{\beta_{\mathrm{I}} t}{2}\right) \frac{\sin \left(\omega_{\mathrm{ad}} t\right)}{\omega_{\mathrm{ad}}}
\end{aligned}
$$

4.1.1. Stability Setting $f(t)=0$ in equation (13), Laplace transforming (13) and (14) with respect to $t$ and combining these equations we have the characteristic equation for the complete system

$$
\begin{aligned}
& s^{4}+\left(\beta_{\mathrm{a}}+\beta_{1}\right) s^{3}+\left(\omega_{\mathrm{a}}^{2}+\omega_{1}^{2}+\beta_{1} \beta_{\mathrm{a}}\right) s^{2}+\left(\omega_{\mathrm{a}}^{2} \beta_{1}+\omega_{1}^{2} \beta_{\mathrm{a}}\right) s \\
& \quad+\omega_{\mathrm{a}}^{2} \omega_{1}^{2}(1-\lambda)=0 \\
& \begin{array}{l}
\text { Which is of the form } \\
\quad s^{4}+a_{1} s^{3}+a_{2} s^{2}+a_{3} s+a_{4}=0
\end{array}
\end{aligned}
$$

Applying the Routh Hurwitz criterion to the characteristic equation, necessary and sufficient conditions for stability 
are:

$$
\begin{aligned}
& \text { (i) } a_{i}>0, i=1,2,3,4 \\
& \text { (ii) } a_{1} a_{2}>a_{3} \\
& \text { (iii) } a_{1} \hat{a}_{2} a_{3}>a_{1}^{2} a_{4}+a_{3}^{2} .
\end{aligned}
$$

It may be shown that conditions (ii) and (iii) in equation (18a) are satisfied if conditions (i) are satisfied. Hence necessary and sufficient conditions for stability are:

$$
\begin{aligned}
& \text { (1) } \beta_{a}>0 \\
& \text { (2) } \beta_{i}>0 \\
& \text { (3) } 0<\lambda<1 \text {. }
\end{aligned}
$$

Since the inherent system damping is always positive, and since $\beta_{\mathrm{a}}$ is designed to be positive, the only condition for stability is that $\lambda$ be less than unity. Thus if the system is statically stable it is also Liapunov asymptotically stable (LAS). (It should be noted from equations (10) and (11) that static stability requires that $k_{1}>k_{\mathrm{c}}$, or $k_{1}(1-\lambda)>0$, thus if $\lambda>0$, the condition for static stability is that $\lambda<1$.)

4.1.2. Frequency response of system The analysis of the stability of the system under positive position feedback showed that if the combined system was statically stable it was also Liapunov asymptotically stable, and that this was true for any combination of system/actuator frequencies. In this section we shall utilize this property to design a control system which will be robust to changes in the system frequency and damping, which are often not known accurately.

Since the system is LAS all initial displacements and velocities will disappear with time. Thus we need only consider the steady state response to sinusoidal excitation, since by Fourier's Theorem we can synthesis the response to any type of excitation.

Fourier transforming equation (15) with respect to $t$, setting all initial conditions to zero, we have:

$$
\begin{aligned}
& P(\omega) X_{1}(\omega)=Q(\omega) \delta_{1} \omega_{1}^{2} \\
& P(\omega)=\left[\omega^{4}-\left(\omega-a^{2}+\omega_{1}^{2}+\beta_{a} \beta_{1}\right) \omega^{2}+\omega_{\mathrm{a}}^{2} \omega_{1}^{2}(1-\lambda)\right. \\
& \left.\quad-j\left(\beta_{\mathrm{a}}+\beta_{1}\right) \omega^{3}+j\left(\omega_{\mathrm{a}}^{2} \beta_{1}+\omega_{s}^{2} \beta_{\mathrm{a}}\right) \omega\right] \\
& Q(\omega)=\left[\omega_{\mathrm{a}}^{2}-\omega^{2}+j \omega \beta_{\mathrm{a}}\right]
\end{aligned}
$$

where $\delta_{1}=|f| / k_{1}$

Thus the frequency response function $R(\omega)$ is given by:

$$
R_{1}(\omega)=\left|X_{\mathrm{I}}(\omega)\right| / \delta_{1}=\omega_{1}^{2} \frac{|Q(\omega)|}{|P(\omega)|} .
$$

Introducing the dimensionless frequencies $\eta_{n}=\omega_{n} / \omega_{1}$

$$
\begin{aligned}
& R_{\mathrm{I}}(\eta)=\sqrt{\left[\left(\eta_{\mathrm{a}}^{2}-\eta^{2}\right)^{2}+\left(2 \eta \eta_{\mathrm{a}} z_{\mathrm{a}}\right)^{2}\right]} \\
& \quad\left\{\left[\left(1-\eta^{2}\right)\left(\eta_{\mathrm{a}}^{2}-\eta^{2}\right)-4 z_{1} z_{\mathrm{a}} \eta_{\mathrm{a}} \eta^{2}-\lambda \eta_{\mathrm{a}}^{2}\right]^{2}\right. \\
& \left.+4 \eta^{2}\left[\eta_{\mathrm{a}} z_{\mathrm{a}}\left(1-\eta^{2}\right)+z_{1}\left(\eta_{\mathrm{a}}^{2}-\eta^{2}\right)\right]^{2}\right\}^{-1 / 2}
\end{aligned}
$$

where $z_{n}=\beta_{n} / \omega_{1}^{2}$. Examination of equation (23) shows the following properties:

(1) The static response is determined by $\lambda$.

(2) The peak response is determine primarily by $\eta_{\mathrm{a}}$ and $z_{\mathrm{a}}$, which are properties of the actuator.

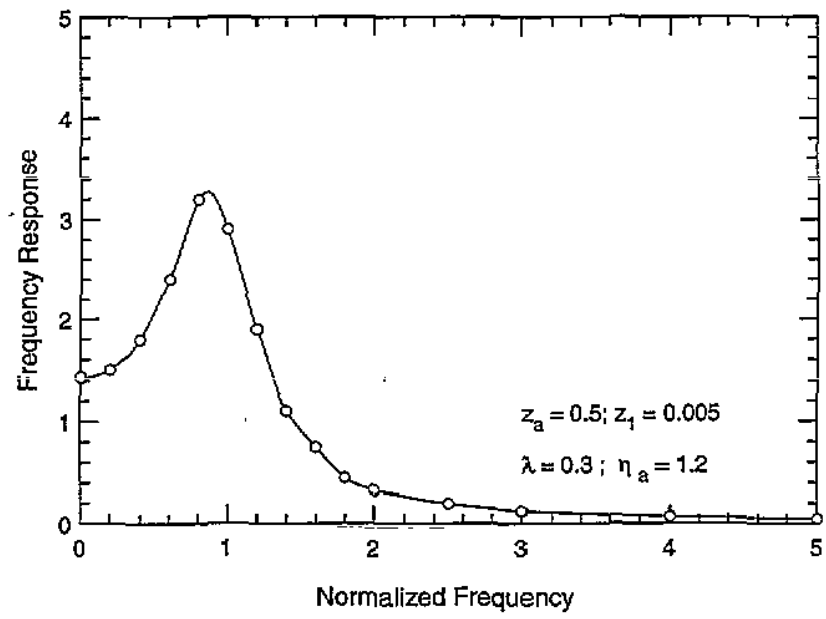

Figure 1.

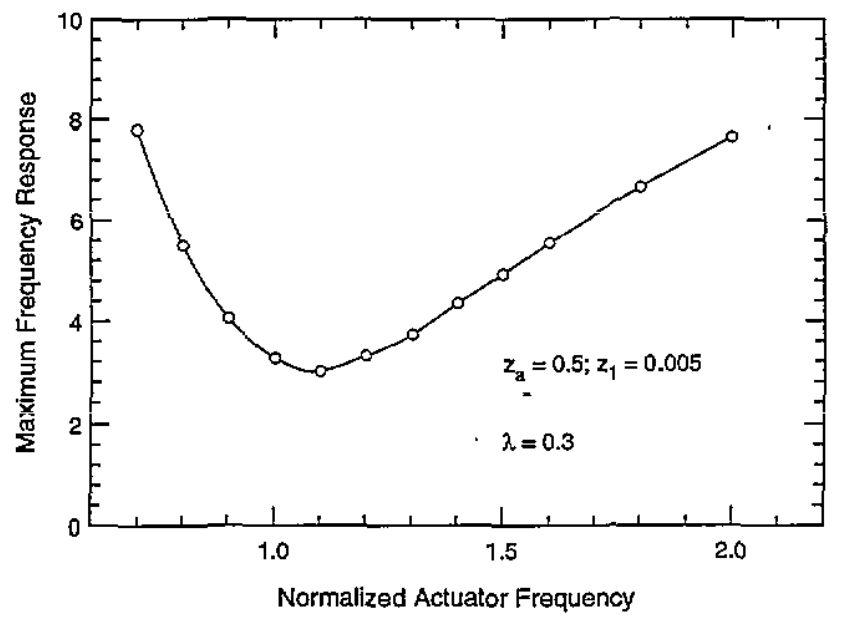

Figure 2.

(3) The intermediate-frequency response is mainly determined by $z_{1}$ which depends on the damping inherent in the system.

(4) The high-frequency response goes to zero as $\eta^{-2}$.

Figure 1 shows the frequency response of the controlled system as a function of $\eta_{\mathrm{a}}$ the normalized natural frequency of the actuator. The values of the damping parameters $z_{a}$ and $z_{1}$ have been set at 0.5 and 0.005 respectively. Figure 2 shows the maximum response of the system for different values of the actuator frequency parameter $\eta_{\mathrm{a}}$ in the range of 0.7 to 2.0. It is seen that best performance is achieved when the actuator is 'tuned' to the vicinity of the system natural frequency, however, the tuning is not very sensitive.

\subsection{Multi degree of freedom case}

Consider now the application of positive position feedback to a discrete multi degree of freedom system in which each spring is equiped with a relative displacement sensor/force actuator pair which can apply a force across the spring proportional to the force through the spring. Furthermore suppose that each actuator has the same dynamic response. 
The equations of motion of such a system are:

$$
\mathrm{M} \ddot{x}+\mathrm{B} \dot{x}+\mathrm{K} \mathcal{H} x=f(x, t)
$$

where: $\mathbf{M}, \mathbf{B}, \mathbf{K}$ are symmmetric positive definite $N$ by $N$ matrices, (in the aerospace context only $M$ is positive definite), $x$ is an $N$ vector and $\mathcal{H}$ is the hereditary operator $\mathcal{H}=\left(1-\lambda \mathcal{H}_{\mathrm{a}}\right)$ where:

$$
\mathcal{H}_{\mathrm{a}}(.)=\int_{-\infty}^{t} h_{\mathrm{a}}(t-\tau)(.) \mathrm{d} \tau
$$

Let $\mathbf{C} x=b$ be the congruence transformation which diagonalizes $\mathbf{M}, \mathbf{B}, \mathbf{K}$, then equation.(25) is transformed to:

$$
\ddot{b}_{i}+\beta_{i} \dot{b}_{i}+\omega_{i}^{2} \mathcal{H} b_{i}=q_{i}(t), i \in[1, N]
$$

4.2.1. Stability Setting $q_{i}(t)=0$ in equation (26) and Laplace transforming with respect to $t$ we have the characteristic equation for the $i$ th. mode of the complete syștêm

$$
\begin{aligned}
s^{4}+ & \left(\beta_{\mathrm{a}}+\beta_{i}\right) s^{3}+\left(\omega_{\mathrm{a}}^{2}+\omega_{i}^{2}+\beta_{i} \beta_{\mathrm{a}}\right) s^{2} \\
& +\left(\omega_{\mathrm{a}}^{2} \beta_{i}+\omega_{i}^{2} \beta_{\mathrm{a}}\right) s+\omega_{\mathrm{a}}^{2} \omega_{i}^{2}(1-\lambda)=0 \\
& i \in[1, N] .
\end{aligned}
$$

Applying the Routh Hurwitz stability criterion to the characteristic equation (27) as before, it is easily seen that necessary and sufficient conditions for stability are:

$$
\begin{aligned}
& \text { (1) } \beta_{\mathrm{a}}>0 \\
& \text { (2) } \beta_{\mathrm{i}}>=0 \\
& \text { (3) } 0<\lambda<1 \text {. }
\end{aligned}
$$

Since the inherent system damping $\beta_{i}$ is always positive, and since $\beta_{\mathrm{a}}$ is designed to be positive, the only condition for stability is that $\lambda$ be less than unity. This is true for each mode of the complete system. Thus if the system is statically stable it is also Liapunov asymptotically stable.

4.2.2. Frequency response of system The analysis of the stability of the system under positive position feedback showed that if the combined system was statically stable it was also Liapunov asymptotically stable, and that this was true for any combination of system/actuator frequencies. In this section we shall utilize this property to design a system which will be insensitive to disturbancies.

Since the system is LAS all initial displacements and velocities will disappear with time. Thus we need only consider the steady state response to sinusoidal excitation, since by Fourier's Theorem we can synthesis the response to any type of excitation.

Fourier transforming equation (26) with respect to $t$, setting all initial conditions to zero, we have:

$$
\begin{aligned}
& P(\omega) B_{i}(\omega)=Q(\omega) \delta_{1} \omega_{1}^{2} \\
& P(\omega)=\left[\omega^{4}-\left(\omega_{\mathrm{a}}^{2}+\omega_{i}^{2}+\beta_{\mathrm{a}} \beta_{i}\right) \omega^{2}+\omega_{\mathrm{a}}^{2} \omega_{i}^{2}(1-\lambda)\right. \\
& \left.\quad-j\left(\beta_{\mathrm{a}}+\beta_{i}\right) \omega^{3}+j\left(\omega_{\mathrm{a}}^{2} \beta_{i}+\omega_{i}^{2} \beta_{\mathrm{a}}\right) \omega\right] \\
& Q(\omega)=\left[\omega_{\mathrm{a}}^{2}-\omega^{2}+j \omega \beta_{\mathrm{a}}\right]
\end{aligned}
$$

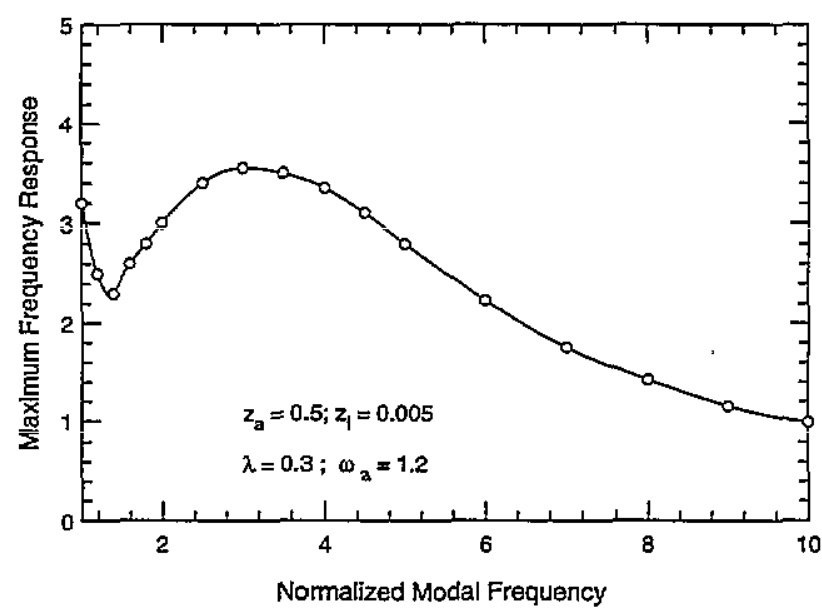

Figure 3.

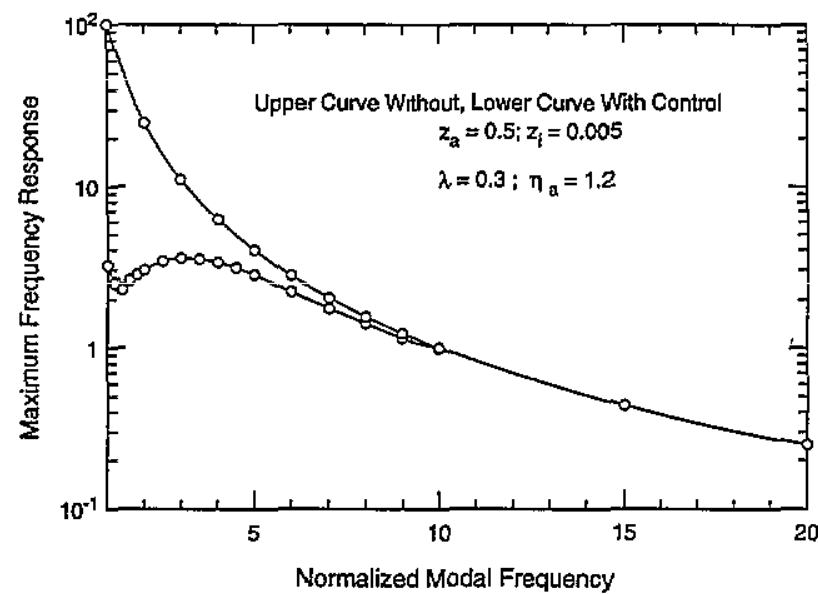

Figure 4.

where $\delta_{1}=|q| / \omega_{1}^{2}$. Thus the frequency response function $R(\omega)$ is given by:

$$
R_{i}(\omega)=\left|B_{i}(\omega)\right| / s_{1}=\omega_{1}^{2} \frac{|Q(\omega)|}{|P(\omega)|}
$$

Introducing the dimensionless frequencies $\eta_{i}=\omega_{i} / \omega_{1}$, and setting $\beta_{\mathrm{a}}=2 \omega_{\mathrm{a}} z_{\mathrm{a}}, \beta_{i}=2 \omega_{i} z_{i}$.

$$
\begin{aligned}
& R_{i}(\eta)=\sqrt{\left[\left(\eta_{\mathrm{a}}^{2}-\eta^{2}\right)^{2}+\left(2 \eta \eta_{\mathrm{a}} z_{\mathrm{a}}\right)^{2}\right]} \\
& \quad\left\{\left[\left(\eta_{i}^{2}-\eta^{2}\right)\left(\eta_{\mathrm{a}}^{2}-\eta^{2}\right)-4 z_{i} z_{\mathrm{a}} z_{i} \eta_{\mathrm{a}} \eta_{i} \eta^{2}-\lambda \eta_{\mathrm{a}}^{2} \eta_{i}^{2}\right]^{2}\right. \\
& \left.\quad+4 \eta^{2}\left[z_{\mathrm{a}} \eta_{\mathrm{a}}\left(\eta_{i}^{2}-\eta^{2}\right)+z_{i} \eta_{i}\left(\eta_{\mathrm{a}}^{2}-\eta^{2}\right)\right]^{2}\right\} .
\end{aligned}
$$

Examination of equation (33) shows the following properties

(1) The static response is determined by $\lambda$.

(2) The peak response is determine primarily by $\eta_{\mathrm{a}}$ and $z_{\mathrm{a}}$, which are properties of the actuator.

(3) The intermediate-frequency response is mainly determined by $z_{i}$ which depends on the damping inherent in the system.

(4) The high-frequency response tends to zero as $\eta^{-2}$.

Figure 3 shows the peak-frequency response of the eigenmodes of the controlled system for different values 
of $\eta_{i}$ the normalized natural frequency of the $i$ th. mode. The values of the parameters $\omega_{\mathrm{a}}, z_{\mathrm{a}}$ and $z_{i}$ have been set at $1.2,0.5$ and 0.005 respectively. It is seen that the peak response in all modes is less than 3.2. Figure 4 shows a comparison of the peak response in all modes with and without control. It is seen that the response in all modes is less than or equal to the response without control. In particular the response of the first mode is reduced by a factor of over thirty, if the internal damping, in the system is only 0.001 then the response in the first mode is reduced by a factor of over one hundred and fifty.

\subsubsection{Continuous systems Fanson and Caughey (1987)} applied the concept of positive position feedback to the vibrational control of a cantilevered beam using a single collocated piezoelectric sensor/actuator pair. By using tuned filters in the feedback loop excellent control was obtained for the first five modes of vibration. Dosch et al (1992) showed that one could combine the piezoelectric sensor/ actuator in a single element. This raises the possibility that with modern micro- fabrication techniques one could make engineering materials in which the sensor/actuator elements and the associated amplifiers were incorporated into the materials. Structures fabricated from such materials would have highly desirable properties. To illustrate these ideas, consider the following model problem, of a simply supported beam fabricated out of such a 'smart' material

$\Pi U_{t t}-\Delta U_{x x t}+\Gamma \mathcal{H} U_{x x x x}=f(x, t) \quad 0<x<L$

With simply supported boundary conditions, the eigenfunctions for equation (34) are:

$$
\Phi_{i}(x)=\sqrt{2 /(\Pi L)} \sin (\pi i x / L) \quad i \in[1, \infty) .
$$

The corresponding eigenvalues are:

$$
\omega_{i}^{2}=(i \pi / L)^{2} \sqrt{\Gamma / \Pi}, i \in[1, \infty) .
$$

Expressing the solution of equation (34) in the form:

$$
U(x, t)=\sum_{i=0}^{\infty} a_{i}(t) \Phi_{i}(x)
$$

Substituting equation (37) into equation (35) and making use of the orthogonality of the eigenfunctions we have:

$$
\ddot{a}_{i}+2 \omega_{i} z_{i} \dot{a}_{i}+\omega_{i}^{2} \mathcal{H} a_{i}=q_{i}(t)
$$

where:

$$
q_{i}(t)=\int_{0}^{L} \Phi_{i}(x) f(x, t) \mathrm{d} x .
$$

The structure of equation (38) is identical in form to that of equation (26) except that $N=\infty$. Thus we see that all the eigen modes of the continuous system are Liapunov asymptotically stable for $\lambda<1$, hence if the system is statically stable it is also LAS. Furthermore, except for the fact that the natural frequencies extend to infinity, the frequency response of the continuous system are given by equation (33). Therefore if $z_{i}=z=0.005$, and $z_{\mathrm{a}}$ in $\mathcal{H}_{\mathrm{a}}=0.5$, then the response of the continuous system can be obtained from Figures 3 and 4. Thus we see that for continuous systems 'smart' material implimentation of positive position feedback gives a control system which is robust to variations in the frequecies and damping of the eigen modes, which are usually not known accurately.

An alternative derivation of the stabilitity of the controlled beam is established in Appendix A without the use of eigenfunctions.

It should be noted that in the case of discrete multidegree-of-freedom systems, if the 'springs' are fabricated from 'smart' material, the system will automatically have the proper form for positive position feedback control.

\section{Conclusions}

(1) It has been shown that the dynamic analysis of structures fabricated from a homogeneous 'smart' material is greatly simplified by the observation that the eigen functions of such structures are identical to those of the same structure fabricated entirely of purely elastic materials. The dynamic analysis of such structures is thus reduced to the temporal analysis of the eigenmodes of the structure.

(2) It has been shown that 'positive position feedback control' is easily implimented in concept in both discrete and continuous systems. It is shown that one can design such a control system which will significantly increase the damping in the lower-frequency modes of a structure without affecting the stability of the uncontrolled higherfrequency, or unmodelled modes. Furthermore it is shown that an exact knowledge of the natural frequencies of the structue is not required in order to design an effective control system. With modern micro-fabrication techniques it is possible to miniaturize the sensors, actuators and amplifiers used in positive position feedback and to construct structural materials which have the desired damping properties built into them.

\section{Appendix A. Stability of positive position feedback for continuous systems}

The stability of the continuous model system equation (34) can be established directly without recourse to the use of eigenfunctions, to this end we can rewrite equation (34) in scaled component form:

$$
\begin{aligned}
& U_{t t}-\beta U_{x x t}+U_{x x x x}=\omega_{\mathrm{a}} \lambda V_{x x} ; 0<x<\pi \\
& V_{t t}+\beta_{\mathrm{a}} V_{t}+\omega_{\mathrm{a}}^{2} V=\omega_{\mathrm{a}} \lambda U_{x x}
\end{aligned}
$$

where $U(x, t)$ is the displacement of the simply supported beam we wish to control, $V(x, t)$ is the control signal, and $\lambda<1$

Consider the Liapunov function $\mathcal{V}(t)$ :

$$
\mathcal{V}(t)=1 / 2 \int_{0}^{\pi}\left[U_{t}^{2}+V_{t}^{2}+U_{x x}^{2}+\omega_{\mathrm{a}}^{2} V^{2}+2 \omega_{\mathrm{a}} \lambda U_{x} V_{x}\right] \mathrm{d} x
$$

Using the Cauchy-Schwartz inequality it is easily seen that $\mathcal{V}(t)$ is positive definite for $U(x, t) \neq 0$.

Differentiating $\mathcal{V}(t)$ with respect to $t$ and evaluating along the trajectories of the motion, we have:

$$
\dot{\mathcal{V}}(t)=-\int_{0}^{\pi}\left[\beta U_{x t}^{2}+\beta_{\mathrm{a}} V_{t}^{2}\right] \mathrm{d} x \leq 0
$$


Thus the system is Liapunov stable, however, standard arguments show that unless $U(x, t)=V(x, t)=0, \dot{V}$ can vanish only on sets of zero measure, thus the controlled beam is in fact Liapunov asymptotically stable.

\section{References}

Aubrun J N 1980 J. Guidance Control 3444

Balas M J 1978a IEEE Trans. Autom. Control 2674

-1978b J. Guidance Control 1 93; 2252

1982 IEEE Trans. Autom. Control 27522

Caughey $\mathrm{T} \overline{\mathrm{K}} 1962$ 4th US National Congress of Applied Mechanics 87

Caughey T K and Goh C J 1982 Dynamics Laboratory Report DYNL 82-3 California Institute of Technology
1983 NASA JPL Publication 83-1 119

Chen C L 1982 Dynamics Laboratory Report DYNL 82-1 California Institute of Technology

Dosch J J, Inman D J and Garcia E $1992 J$. Intelligent Mater. Sys. Struct. 3166

Fanson J L and Caughey T K 1987 AlAA Paper No. 87.0902 588

Fanson $\mathrm{J} \mathrm{L}$ An Experimental Investigation of Vibration Suppression in Large Space Structures Using Positive Position Feedback, Caltech PhD Thesis

Goh C J 1983 Analysis and Control of Quasi Distributed Parameter Systems, Caltech PhD Thesis

Goh, C J and Caughey T K 1985 Int. J. Control 41787

Meirovitch L, Baruh $\mathrm{H}$ and $\mathrm{Oz} \mathrm{H}$ AAS/AIAA Astrodynamics Specialists Conference, Lake Tahoe, Nevada 81

Schaechter D B 1982 J. Guidance Control Dynam. 548 